\title{
Digitalization of processes in livestock farming: software solutions in the case of Bulgaria
}

\author{
Konstantin Stoyanov ${ }^{*}$, George Zhelyazkov ${ }^{1}$, and Nikolay Penev ${ }^{1}$ \\ ${ }^{1}$ Faculty of Economics, Trakia University, Student Campus, Stara Zagora, Bulgaria
}

\begin{abstract}
Digitalization has already been largely seen as a key competitive advantage for all enterprises regardless of their economic sector or field of activity. Traditional economic activities such as agricultural and livestock farming have not been left out from those innovative approaches. The article focuses on some unorthodox economic performances shown by Bulgarian livestock farms in the recent years, including Farm Net Value Added by Annual Work Unit and VAT on investments. The article draws the general framework in terms of available products targeting medium and large enterprises in livestock farming addressing their needs for digitalization and automation of processes. The authors include an overview of existing software solutions for the Bulgarian market. Additional comparative analysis of available products, developed for livestock farming companies, will be drawn, emphasizing on the tools and different options they provide.
\end{abstract}

\section{Introduction}

Livestock and agriculture are one of the traditional sectors that are vital for development of societies and independent national economies. Changes in those sectors, both at structural and organizational level, over different periods of time, are of extreme importance not only for individual regions and countries, but also for the societies in general. It is an indisputable fact that the distribution of goods and the sustainable provision of food has a key role for the independence and development of any country. At the same time, for the last more than 50 years, the planet's population has been growing at a hitherto unknown rate. This creates indisputable conditions for continuous and permanent economic growth, due to the evergrowing number of labour force, but also thanks to the constantly increasing labour productivity, dictated by changes in technology and process optimization in all economic sectors. However, such an important growth in the world's population creates new challenges in terms of accessing limited resources and demands finding new approaches, even in traditional sectors such as livestock and agriculture.

\section{Methodology}

The study uses various analyses of official and secondary databases, reports, surveys, webbased searches.

\footnotetext{
*Corresponding author: konstantin.stoyanov@trakia-uni.bg
} 
The software products, which are discussed further in the article, are a result of an online search conducted within the month of June 2021, as researchers were using keywords written only in Bulgarian. Additionally, the listed companies included in the research are established or have official representations on the Bulgarian market. The online search met the following keyword criteria:

- Livestock software

- Farm software

- Farm process automation software

- Farm management software

- Farm process management software

After identifying appropriate products offering farm management solutions, specific technical specifications, scope, limitations, and possibilities are considered. Based on this, a comparative analysis examines the complexity of individual products and their applicability in view of the processes of modernization and digitalization of livestock farms.

\section{Some observations and main conclusions}

In general, the major economies in developed countries have long taken the step towards automating some of the processes of governance and the entry of digitalization in various sectors and areas of activity. This phenomenon was further exacerbated by the pandemic, which affected the whole world during significant periods of 2020. However, the processes of digitalization are known to be familiar in livestock farming and agriculture. The concept of precision farming is subordinated to the need for optimal allocation of resources, automation of some processes and facilitated decision-making that will lead to maximum results to support enterprises in a competitive sector of the economy.

Livestock farming is largely characterized by 24 -hour periodicity of processes and in its organizational structure is reminiscent of traditional industrial production in terms of loading the machineries and equipment used in the activities on a daily basis. This, in itself, is a prerequisite for the gradual introduction of new technological solutions to facilitate and automate processes, limiting human intervention and gradually implementing the concepts of the fourth industrial revolution in the sector.

These trends, observed for decades mainly in the countries of Western and Northern Europe, are a sufficient prerequisite to be implemented by Bulgarian companies to meet the development of the sector and to be able to compete in a free European market. However, the need for modernization is also dictated by some other macroeconomic factors that are observed in the livestock sector and are typical for Bulgarian enterprises engaged in this type of activity. According to a study by EIT Food [1], there are several obstacles that hinder the transition of the Bulgarian economy to one focused on innovation. Livestock farming, as one of the key sectors in the country's economy, is also affected by these factors, namely:

- Insufficient labour force

- Low levels of investment, both foreign and those aimed at modernization of production processes

- Uncertainty in the implemented policies

- Delay in environmental reforms

The modernization and automation of some of the processes in livestock farms, as mentioned earlier in the article, provide answers, and offer solutions to many of the above challenges. However, it is necessary to consider what specific options exist for businesses that are willing to make the leap. Most of the software systems are aimed primarily at large livestock farms, as their integration will have a tangible effect on their work and financial performance in the foreseeable future. Therefore, the results of this study are aimed primarily at large and medium-sized livestock farms operating on the Bulgarian market. 
There is still not enough comprehensive research to demonstrate whether livestock farms make investments related to the digitalization of processes, what is their size and their effect on the productivity and income of individual enterprises. However, there are some studies and databases that give a broader view of livestock farms in Bulgaria as a whole and their structural and financial condition.

The Farm Accountancy Data Network (FADN), set up in 1965, supports the EU's common agricultural policy by determining the income of farms in the EU and providing farm-level analyses based on standardized microeconomic data collected annually from around 80,000 farms. [2] Some of the data can serve as a basis for deriving some trends and interpreting the behavior and results achieved by livestock farms in Bulgaria. Of particular interest is the possibility to compare them with the average results for the livestock farms in the European Union, which allows an assessment to be made on the basis of situational analysis for the specific study period.

Beyond the traditional financial and economic indicators, which can be linked to various factors, including those related to regional specifics, there are some interesting, unorthodox results. They can offer a different point of view of the situation and offer solutions for improving the activity of livestock farms in Bulgaria.

One of the indicators of interest is Farm Net Value Added. It represents the sum of all factors of production, both farm and external. It is equal to output and subsidies but excluding intermediate consumption and depreciation. This indicator is an important marker of the economic performance of farms. It should be borne in mind that salaries, rents, and interest must be paid from it and own factors must be reimbursed. As can be seen from Figure 1, Bulgaria encounters some significant differences from the EU average in terms of performance on all types of farms, except pig and poultry farms.

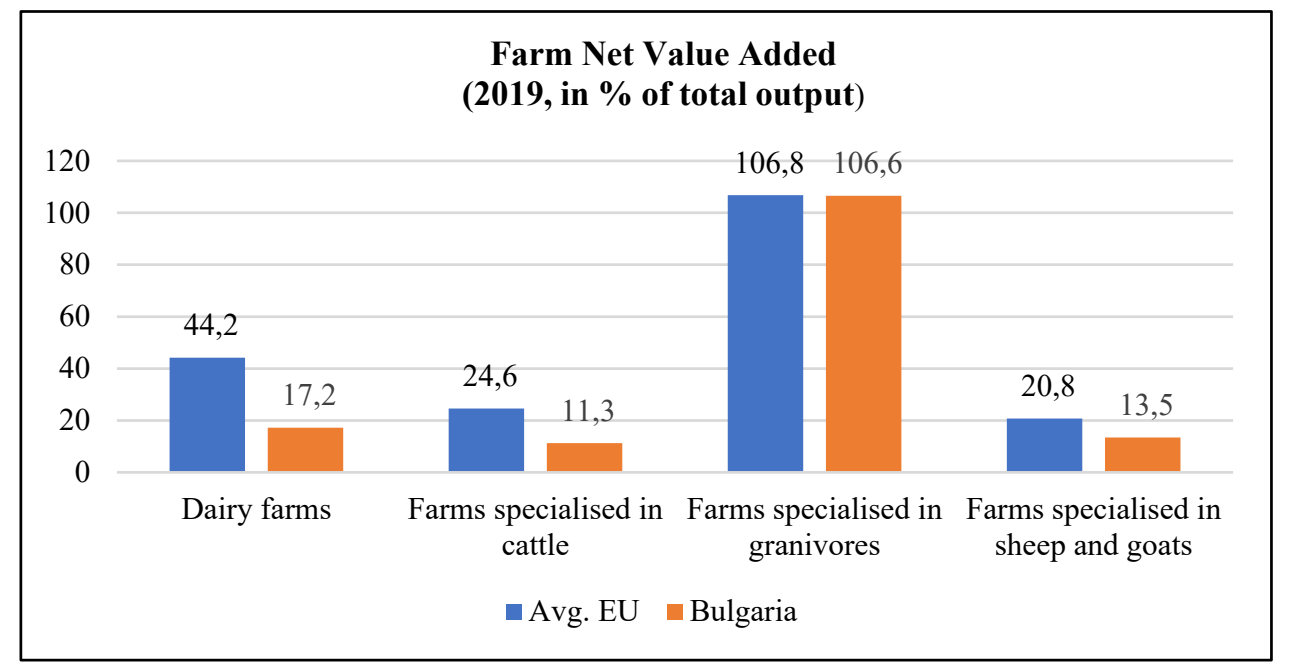

Fig. 1. Bulgarian livestock farms net value added in 2019 compared to the average for EU (Source: Authors' representation based on FADN data)

These differences become even more significant when the added value of the farm is observed relatively to the annual work units. Expressed in this way, the average result of the observed farms can take into account the disparities in the labour market in the different Member States. In this manner, the remuneration costs are already taken into account and what is observed is the value that actually remains on the farm. Figure 2 reveals that contrary to expectations, due to lower average wage levels in Bulgaria, livestock farms in 2019 generate a net value added by annual work unit of less than 10000 EUR, except for pig and 
poultry farms, where this value is of 16000 EUR. In any case, the differences from the EU averages are close and more than 3 times. This indicates lower productivity of Bulgarian livestock farms and a noticeably weaker economic result.

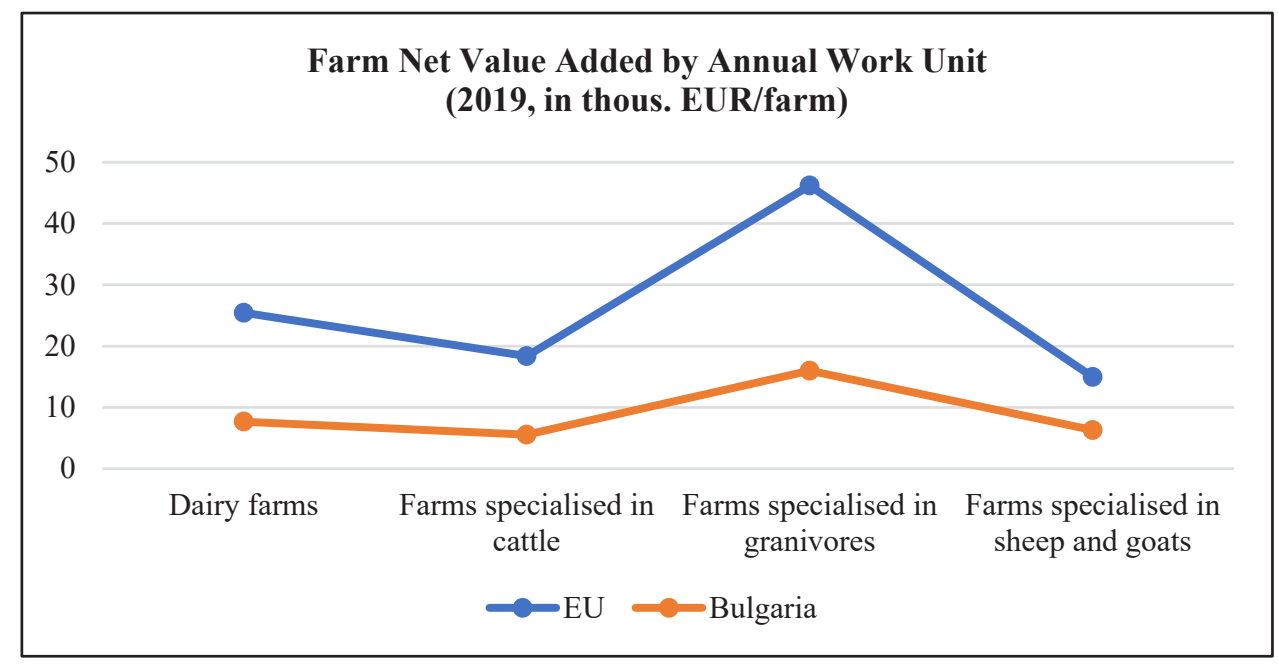

Fig. 2. Bulgarian livestock farms net value added by Annual work unit in 2019 compared to the average for EU

(Source: Authors' representation based on FADN data)

VAT on investments is considered as a separate economic indicator, independent of revenues, as this is usually a significant amount that is not directly related to revenues on an annual basis. However, in the specific case of the present study, investment is a key component for the development of the sector and the establishment of a competitive advantage. As it can be seen in Figure 3, past 2016 the average income per farm unit marked a significant increase, which shall be the subject of a separate study. It can be assumed that the maintenance of higher revenues in the period 2017-2019 is a result of the investments made in 2015. At the same time, the number of observed farms since 2017 falling in the scope of the study is lower, so it is necessary to make additional observations.

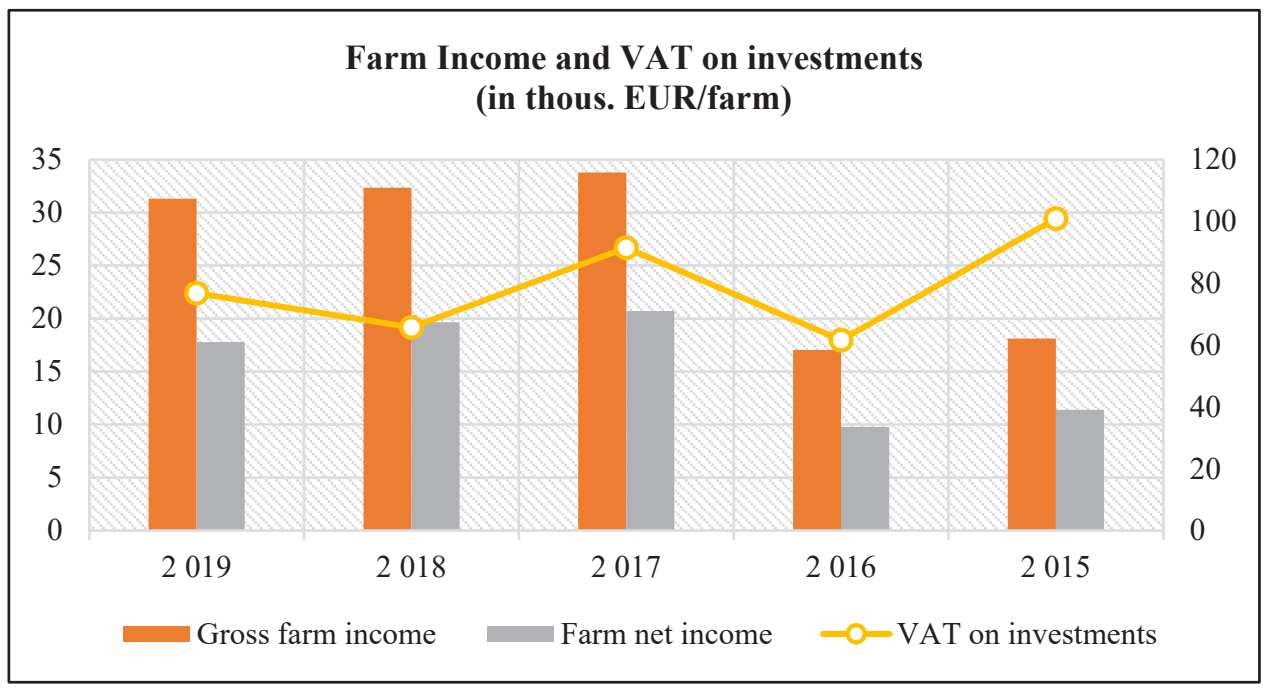

Fig. 3. Bulgarian livestock farms investments, gross and net incomes, 2015-2019 
(Source: Authors' representation based on FADN data)

It is evident from these results that livestock farms in Bulgaria need investments that are aimed both at modernization of farms and the introduction of new technologies and automation of processes, as labour costs take away much of their added value. According to Latruffe [3], there are several different prerequisites for determining the competitiveness of enterprises in the agricultural sector, dividing the main components into those that depend on the internal organizational processes in the company and those that depend on the external environment and are outside the control of companies. For the present study, the role of the variable factors that can be influenced by purposeful actions and decisions in the individual structures is essential. Farm size and social capital are not considerations that would be relevant to the objectives of this study, but structural characteristics can have a positive effect on the technical efficiency of the farm. By investing in new technologies, reflected as debt or indebtedness, livestock farms can register a positive change in their productivity, and hence to register growth in revenue and profit in subsequent periods.

The digitalization of processes and precision agriculture in livestock farming, according to most studies, are aimed at caring for animals, improving their nutrition and health, etc. From an organizational and economic point of view, however, livestock farms could improve their performance in all other aspects of traditional ancillary activities, such as tracking operations, inventory management, financial accounting operations and other management positions. However, in essence, they are all subordinated to the main activity related to livestock farming and the software management products must be adapted to the specific needs.

This characteristic determines the need for specialized software that is applicable to the needs of livestock farms. In the last 10 years, many solutions can be found on the world market, providing complete management of the activities on the farm.

Table 1 examines the products that can be found on the Bulgarian market and offer support in Bulgarian, which is important for local consumers. The main criterion for their inclusion in this study is the provision of an option to manage some of the processes that are related to managerial decision making, financial management and other similar operations.

Table 1. Software products for livestock farms management in Bulgaria

\begin{tabular}{|c|c|c|c|c|c|}
\hline Software product & $\begin{array}{c}\text { Developed for } \\
\text { livestock farms }\end{array}$ & $\begin{array}{c}\text { Modular } \\
\text { system }\end{array}$ & $\begin{array}{c}\text { Operational } \\
\text { Module }\end{array}$ & $\begin{array}{c}\text { Financial } \\
\text { Module }\end{array}$ & $\begin{array}{c}\text { Managarial } \\
\text { Module }\end{array}$ \\
\hline Agrosistemi [4] & Yes & Yes & Yes & Yes & Yes \\
\hline Ferma WEB [5] & Yes & Yes & Yes & Yes & Yes \\
\hline TechnoFarm [6] & No & Yes & Yes & No & Yes \\
\hline Acstre [7] & Yes & No & Yes & No & Yes \\
\hline Atlantiify [8] & No & Yes & Yes & No & Yes \\
\hline $\begin{array}{c}\text { Farm Technology } \\
{[9]}\end{array}$ & Yes & Yes & Yes & No & No \\
\hline $\begin{array}{c}\text { DeLaval DelPro } \\
\text { (Agritop) [10] }\end{array}$ & Yes & Yes & Yes & No & No \\
\hline $\begin{array}{c}\text { Big Dutchman } \\
{[11]}\end{array}$ & Yes & Yes & Yes & Yes & Yes \\
\hline
\end{tabular}

Some of the available products are rather targeted at agricultural farming enterprises. They are to a large extent related to the need for larger-scale observations of the areas and analysis of the resulting data. Despite these specifics, some of the products can also be used by livestock farming companies. These products do not offer a full range of options, as mentioned above, but are aimed at modernizing farms and digitalizing some of the processes and are also of potential interest for the managers of livestock farms. 
Depending on the needs and type of livestock farms, different solutions can be found on the market. It is indisputable that in the coming years the introduction of software solutions for comprehensive farm management will become increasingly important. The entry of all sectors of the economy in the conditions of digital process management will bring with it key competitive advantages, even in traditional industries such as livestock farming and agriculture.

There is a need to deepen research in this area and to clearly trace some measurables, including financial, benefits of digitalizing farm management processes. Of additional interest are other innovations that will affect this sector, including blockchain technologies, which shall open up new opportunities for livestock farming enterprises.

The research leading to these results has received funding from the Ministry of education and science under the National science program INTELLIGENT ANIMAL HUSBANDRY, grant agreement $\mathrm{n}^{\circ}$ Д01-62/18.03.2021

\section{References}

1. Food Foresight, Impact of COVID-19 on the agri-food sector in Central and Eastern Europe. Country report: Bulgaria, (2020)

2. European Commission, Farm accountancy data network, https://ec.europa.eu/info/foodfarming-fisheries/farming/facts-and-figures/farms-farming-and-innovation/structures-andeconomics/economics/fadn_en

3. L. Latruffe, OECD Food, Agriculture and Fisheries Working Papers, No. 30, (2010)

4. Official website of Agrosystems.bg, , https://agrosystems-bg.com/bg/moduli/ferma

5. Official website of Fermawebhttp://fermaweb.com/bg

6. Official website of Technofarm, https://www.technofarm.bg/

7. Official website of ACSTRE Software Group https://acstre.com/subsection-75specializiran_softue.html

8. Official website of Atlantify, https:/www.atlantify.com/bg/индустрии/софтуер-заселското-стопанство

9. Official website of Farmtech, http://farmtech.bg/

10. Official website of Agritop, https://agritop.bg/bg/Upravlenie_na_stado_DeLaval-c138

11. Official website of BigDutchman, https://www.bigdutchman.bg/bg/kompanija/zanas/za-nas/ 